Revue Française de Civilisation Britannique

\title{
Effet Brexit, effet Corbyn ou crise existentielle ? Les dimensions de l'échec travailliste
}

Brexit Effect, Corbyn Effect or Existential Crisis? The Dimensions of the Labour Party's Electoral Failure

\section{Gilles Leydier}

\section{OpenEdition Journals}

\section{Édition électronique}

URL : http://journals.openedition.org/rfcb/5738

DOI : $10.4000 /$ rfcb. 5738

ISSN : 2429-4373

Éditeur

CRECIB - Centre de recherche et d'études en civilisation britannique

\section{Référence électronique}

Gilles Leydier, «Effet Brexit, effet Corbyn ou crise existentielle ? Les dimensions de l'échec travailliste », Revue Française de Civilisation Britannique [En ligne], XXV-3 | 2020, mis en ligne le 10 septembre 2020, consulté le 10 septembre 2020. URL : http://journals.openedition.org/rfcb/5738 ; DOl : https://doi.org/10.4000/rfcb.5738

Ce document a été généré automatiquement le 10 septembre 2020.

\section{cc)}

Revue française de civilisation britannique est mis à disposition selon les termes de la licence Creative Commons Attribution - Pas d'Utilisation Commerciale - Pas de Modification 4.0 International. 


\title{
Effet Brexit, effet Corbyn ou crise existentielle? Les dimensions de l'échec travailliste
}

\author{
Brexit Effect, Corbyn Effect or Existential Crisis? The Dimensions of the Labour \\ Party's Electoral Failure
}

Gilles Leydier

\section{Introduction}

1 "Collapse ", "calamity", "catastrophy", "disaster ", "nightmare ", "humiliation", "crushing emotional blow», «total repudiation », "existential crisis » ${ }^{1} .$. Ces mots, relayés dans la presse et exprimés par les élus, militants, sympathisants ou commentateurs proches du parti travailliste au lendemain du 12 décembre 2019, traduisent un désarroi profond, à la mesure de l'échec retentissant du Labour à l'occasion des dernières élections britanniques.

2 Le scrutin législatif a en effet constitué pour les Travaillistes britanniques une défaite spectaculaire. Ce résultat a pu paraitre surprenant, car survenant deux ans seulement après une précédente consultation relativement serrée - $40 \%$ des voix pour le Labour, contre $42,4 \%$ pour les Tories - et qui avait été notamment caractérisée par la dynamique positive du parti travailliste et de son leader Jeremy Corbyn. Mais il est surtout cruel - notamment en raison de la perte de nombreux sièges symboliques historiquement fidèles au Labour - et fondamentalement préoccupant, de par l'ampleur et le caractère répétitif de la défaite subie. Après 2010, 2015 et 2017, le parti travailliste a ainsi concédé une quatrième défaite de rang face à un parti Conservateur qui, au pouvoir depuis une décennie, a pour sa part réussi à augmenter son score électoral à chaque consultation, et ce dans un contexte d'austérité budgétaire et de politiques sociales restrictives susceptibles de porter un mécontentement populaire favorable à l'opposition travailliste. 
3 Par rapport aux précédentes élections de 2017, le Labour a ainsi perdu 2,5 millions d'électeurs - passant de 12,8 à 10,3 millions - vu son score chuter de $40 \%$ à $32,2 \%$ - soit un différentiel de plus de 11 points par rapport aux 43,6\% obtenus par les Conservateurs - et a perdu une soixantaine de sièges de députés - presque exclusivement au profit du parti de Boris Johnson - pour atteindre le niveau exceptionnellement bas de 203 MPs - soit moins d'un tiers des 650 que compte la chambre des communes.

\section{Mise en perspective historique}

4 Si l'on replace ces chiffres dans une perspective à long terme, le scrutin de 2019 a été marqué pour le parti de Jeremy Corbyn par un résultat historiquement faible si l'on prend pour référence le nombre de voix et/ou le pourcentage de suffrages exprimés. Ainsi avec moins d'un tiers des suffrages, le Labour obtient un de ses scores les plus décevants depuis 1945 (cf. Tableau 1).

Tableau 1 : Le \% de voix obtenu par le parti travailliste aux élections législatives depuis 1945

\begin{tabular}{|l|l|l|l|l|l|l|l|l|l|l|l|}
\hline Elections & $\mathbf{1 9 4 5}$ & $\mathbf{1 9 5 0}$ & $\mathbf{1 9 5 1}$ & $\mathbf{1 9 5 5}$ & $\mathbf{1 9 5 9}$ & $\mathbf{1 9 6 4}$ & $\mathbf{1 9 6 6}$ & $\mathbf{1 9 7 0}$ & $\mathbf{F 1 9 7 4}$ & $\mathbf{0 1 9 7 4}$ & $\mathbf{1 9 7 9}$ \\
\hline \% voix & 47,8 & 46,1 & 48,8 & 46,4 & 43,8 & 44,1 & 47,9 & 43,0 & 37,1 & 39,2 & 36,9 \\
\hline
\end{tabular}

\begin{tabular}{|l|c|c|c|c|c|c|c|c|c|c|}
\hline Elections & 1983 & 1987 & 1992 & 1997 & 2001 & 2005 & 2010 & 2015 & 2017 & 2019 \\
\hline \% voix & 27,7 & 30,8 & 34,4 & 43,2 & 40,7 & 35,3 & 29,0 & 30,4 & 40,0 & 32,2 \\
\hline
\end{tabular}

Source : BBC news, 2019

5 Pour trouver trace d'un résultat plus médiocre, il faut remonter aux années 1980 et aux scrutins de 1983 et 1987 - tenus dans un contexte politique particulier, marqué par la scission au sein des Travaillistes avec la création du SDP et la concurrence électorale de l'Alliance libérale/SDP - ou aux consultations plus récentes de 2010 et 2015. Remarquons donc que si le score travailliste obtenu en 2019 est historiquement faible, c'est surtout par comparaison avec les résultats obtenus dans les années du post-war consensus (1945-1974) ou pendant la période dominée par le New Labour de Tony Blair (1997-2005). Par contre ce score autour de 32\% correspond exactement à la moyenne du score obtenu par le Labour pendant les années de thatchérisme triomphant (1983-1992), ainsi que depuis l'arrivée au pouvoir d'une majorité conservatrice à partir de 2010, sous les leaderships successifs de David Cameron, Theresa May puis Boris Johnson. En conséquence, avec le recul, c'est le précédent scrutin de 2017 qui apparaît rétrospectivement comme une exception électorale, plutôt que celui de 2019. Ce dernier s'inscrit pleinement dans une tendance au déclin de l'attractivité du Labour, ainsi que de difficultés structurelles de ce dernier dans une situation d'opposition prolongée, associée à un contexte général d'affaiblissement du bipartisme, une situation sur ce dernier point bien différente de celle des années 1951-1964. Si l'on analyse les résultats en termes de représentation électorale, la défaite électorale de 2019 apparait plus sévère encore pour les Travaillistes (cf. Tableau 2). 
Tableau 2 : Le nombre de sièges de députés obtenu par le parti travailliste aux élections législatives depuis 1935

\begin{tabular}{|l|l|l|l|l|l|l|l|l|l|l|l|l|}
\hline Elections & 1935 & 1945 & 1950 & 1951 & 1955 & 1959 & 1964 & 1966 & 1970 & F74 & 074 & 1979 \\
\hline \% sièges & 154 & 393 & 315 & 295 & 277 & 258 & 317 & 363 & 287 & 301 & 319 & 269 \\
\hline
\end{tabular}

\begin{tabular}{|l|l|l|l|l|l|l|l|l|l|l|}
\hline Elections & 1983 & 1987 & 1992 & 1997 & 2001 & 2005 & 2010 & 2015 & 2017 & 2019 \\
\hline \% sièges & 209 & 229 & 271 & 419 & 413 & 356 & 258 & 232 & 262 & 203 \\
\hline
\end{tabular}

Source : BBC news

6 Avec seulement 203 députés, il faut en effet remonter avant la Seconde Guerre mondiale et aux législatives de 1935 pour trouver la trace d'un nombre d'élus aux Communes plus faible. Et par rapport à sa moyenne basse enregistrée pendant les années 1980 ou au cours de la décennie précédente, le Labour est affaibli par la perte d'une grosse quarantaine de sièges de députés.

7 L'accentuation de l'échec électoral du Labour en termes de représentation est notamment liée à l'effondrement récent de sa base écossaise, qui avait apporté au groupe parlementaire travailliste de Westminster au moins 40 sièges de députés à chaque élection entre 1964 et 2010.

\section{Les dimensions de la défaite}

8 La baisse d'attractivité du parti travailliste en 2019 apparait particulièrement spectaculaire si l'on s'intéresse à la cartographie électorale ainsi qu'aux évolutions du comportement électoral dans les différentes catégories sociales (cf. Tableau 3).

Tableau 3 : L'évolution du score comparé des Travaillistes et des Conservateurs dans les différentes catégories de l'électorat entre les élections de 2017 et de 2019

\begin{tabular}{|l|l|l|l|l|l|l|l|}
\hline \% voix & Total & $\mathbf{1 8 - 2 4}$ & $\mathbf{2 5 - 3 4}$ & $\mathbf{3 5 - 4 4}$ & $\mathbf{4 5 - 5 4}$ & $\mathbf{5 5 - 6 4}$ & $\mathbf{6 5 +}$ \\
\hline Travaillistes 2019 & 33 & 57 & 55 & 45 & 35 & 27 & 18 \\
\hline Travaillistes 2017 & 39 & 67 & 58 & 50 & 39 & 33 & 23 \\
\hline Conservateurs 2019 & 45 & 19 & 23 & 30 & 43 & 49 & 62 \\
\hline Conservateurs 2017 & 41 & 18 & 22 & 30 & 40 & 47 & 59 \\
\hline
\end{tabular}

\begin{tabular}{|l|l|l|l|l|l|l|l|}
\hline \% voix & Total & H & F & AB & C1 & C2 & DE \\
\hline Travaillistes 2019 & 33 & 29 & 36 & 31 & 33 & 30 & 37 \\
\hline Travaillistes 2017 & 39 & 35 & 42 & 35 & 41 & 39 & 46 \\
\hline
\end{tabular}




\begin{tabular}{|l|l|l|l|l|l|l|l|}
\hline Conservateurs 2019 & 45 & 48 & 42 & 44 & 45 & 50 & 43 \\
\hline Conservateurs 2017 & 41 & 43 & 40 & 44 & 41 & 44 & 34 \\
\hline
\end{tabular}

Source : Lord Ashcroft polls 2019

9 Par rapport à 2017 la baisse de la popularité touche l'ensemble de l'électorat, tous sexes, âges et catégories sociales confondus. L'électorat féminin par exemple, qui avait voté légèrement plus travailliste que conservateur en 2017, a cette fois donné nettement sa préférence au parti de Boris Johnson. Il en est de même pour les catégories en bas de l'échelle sociale (DE), qui avaient largement soutenu les Travaillistes en 2017 par rapport aux Conservateurs (+12 points d'écart pour le Labour) et qui en 2019 ont beaucoup délaissé le parti de Corbyn pour se tourner vers celui de Johnson (+6 pts d'écart).

10 De fait la chute des Travaillistes entre 2017 et 2019 apparait particulièrement spectaculaire chez l'électorat le plus jeune - moins 10 points chez les 18-24 ans, sans que les Conservateurs n'en profitent réellement - et auprès des catégories sociales intermédiaires ( $\mathrm{C} 1$ ) et les moins aisées ( $\mathrm{C} 2$ et $\mathrm{DE}$ ). Ainsi chez les $\mathrm{C} 2$ alors que l'écart entre Travaillistes et Conservateurs était de 5 points en 2017 à l'avantage des Conservateurs, le différentiel est passé à 20 points en faveur des Conservateurs en 2019. Si toutes les catégories de l'électorat ont fait moins confiance au Labour en 2019 par rapport à 2017, le swing des Travaillistes vers les Conservateurs a été particulièrement fort dans les catégories populaires.

$11 \mathrm{Au}$ vu de ces résultats très décevants le parti travailliste peut certes mettre en avant son avance globale auprès des catégories les plus jeunes de la population. En effet l'électorat britannique en 2019 apparait tout autant qu'en 2017 divisé par le facteur générationnel. Si les plus de 45 ans votent majoritairement conservateur - le soutien au parti conservateur augmentant avec l'âge - les 18-45 ans soutiennent plus volontiers le parti travailliste - et ce d'autant plus que l'âge diminue ${ }^{2}$. Et d'autre part le niveau éducationnel joue désormais clairement en faveur des Travaillistes: ceux-ci l'emportent largement dans l'électorat diplômé - $43 \%$ pour le Labour contre $29 \%$ pour les Conservateurs - alors qu'ils sont nettement devancés par les Conservateurs dans l'électorat de niveau intermédiaire - $31 \%$ contre $48 \%$ - et très largement à la traine parmi les votants possédant un niveau faible, inférieur ou égal au GCSE - $25 \%$ pour les Travaillistes, contre $58 \%$ soit pratiquement trois fois plus pour les Conservateurs ${ }^{3}$.

Par ailleurs au plan territorial le parti travailliste peut toujours compter sur des zones de force situées dans les grandes concentrations urbaines - le Labour a ainsi remporté tous les sièges à Bradford, Bristol, Cardiff, Leeds, Liverpool, Manchester, Newcastle, Nottingham et Sheffield, et huit sur neuf à Birmingham - dans les villes universitaires ainsi que dans la capitale britannique, où les Travaillistes ont conservé 49 de leurs 73 sièges du Greater London ${ }^{4}$. 


\section{Pays de Galles, Ecosse, 'Red wall' anglais : Recul, effondrement ou basculement dans trois bastions traditionnels}

13 Mais la géographie électorale de 2019 a surtout été marquée par un recul plus ou moins prononcé et/ou inattendu du vote travailliste dans trois de ses bastions traditionnels, respectivement au pays de Galles, en Ecosse et dans le nord de l'Angleterre.

$14 \mathrm{Au}$ pays de Galles, place forte historique du Labour, ce dernier a certes conservé sa première place avec 22 sièges de députés sur 40 , mais il a concédé un swing de huit points environ au profit du parti conservateur, et il a perdu six sièges, tous au profit du parti de Boris Johnson, qui le talonne désormais en termes de suffrages obtenus $(36,1 \%$ pour les Conservateurs contre $40,9 \%$ pour les Travaillistes) ${ }^{5}$. A la suite de ces élections le modèle partisan gallois pour les élections à Westminster apparait comme un jeu à quatre partis, dominé par un duopole constitué par le couple Travaillistes/ Conservateurs et complété par deux partis d'importance moindre - Nationalistes de Plaid Cymru et Libéraux-Démocrates - plutôt que par la configuration traditionnelle articulée autour d'un parti dominant, à savoir les Travaillistes.

Les résultats du Labour sont bien pires en Ecosse ${ }^{6}$. Dans ce territoire largement dominé par les Travaillistes à partir de la fin des années 1960 et pendant environ un demisiècle, le parti de Jeremy Corbyn semble avoir franchi un nouveau pallier dans son affaiblissement électoral entamé en 2015. Avec à peine 18,6\% des suffrages, le Labour réalise un score environ deux fois moindre que ses plus médiocres résultats d'aprèsguerre (1974 et 1983) et avec un seul siège de député sur 59, il égale son pire total de 2015. Alors que les Travaillistes comptaient encore 41 sièges en 2010, leur résultat les place largement en troisième position, très loin du Scottish National Party $-45 \%$ des suffrages et plus de $80 \%$ des sièges - et relativement à distance des Conservateurs - un quart des suffrages et six sièges - qui apparaissent désormais comme l'opposition la plus crédible aux nationalistes dominants. De fait depuis la fin des années Brown et l'avènement d'une période correspondant à l'installation d'une majorité conservatrice à Westminster et d'une majorité SNP au Parlement écossais de Holyrood, et par ailleurs dominée par le clivage nationaliste-unioniste et les débats autour de l'avenir institutionnel de l'Ecosse, l'électorat a très fortement diminué son soutien au Labour. Et depuis 2015 le différentiel entre le vote travailliste enregistré en Ecosse et celui en Angleterre est très significatif et en progression, au détriment de l'Ecosse (cf. Tableau $4)^{7}$.

Tableau 4 : Le différentiel entre le vote travailliste en Ecosse et en Angleterre aux élections législatives depuis 1945

\begin{tabular}{|l|l|l|l|l|l|l|l|l|l|l|l|}
\hline Elections & 1945 & $\mathbf{1 9 5 0}$ & $\mathbf{1 9 5 1}$ & $\mathbf{1 9 5 5}$ & $\mathbf{1 9 5 9}$ & $\mathbf{1 9 6 4}$ & $\mathbf{1 9 6 6}$ & $\mathbf{1 9 7 0}$ & $\mathbf{F 1 9 7 4}$ & $\mathbf{0 1 9 7 4}$ & $\mathbf{1 9 7 9}$ \\
\hline $\begin{array}{l}\text { Différence } \\
\text { Ecosse-Angleterre }\end{array}$ & $-0,9$ & 0 & $-0,9$ & $-0,1$ & 3,1 & 5,2 & 1,9 & 1,9 & $-1,0$ & $-3,8$ & 4,9 \\
\hline
\end{tabular}

\begin{tabular}{|l|c|c|c|c|c|c|c|c|c|c|}
\hline Elections & 1983 & 1987 & 1992 & 1997 & 2001 & 2005 & 2010 & 2015 & 2017 & 2019 \\
\hline
\end{tabular}




\begin{tabular}{|l|c|c|c|c|c|c|c|c|c|c|}
\hline $\begin{array}{l}\text { Différence } \\
\text { Ecosse-Angleterre }\end{array}$ & 8,2 & 12,9 & 4,3 & 2,0 & 2,5 & 4,1 & 14,2 & $-7,3$ & $-14,8$ & $-15,5$ \\
\hline
\end{tabular}

Source : David McCrone, Center on Constitutional Change, 2019 autre déconvenue - plus douloureuse sans doute - en Angleterre, dans certains de ses bastions historiques du nord, du nord-est et des Midlands ${ }^{8}$. Si la désillusion écossaise était plutôt anticipée, la soirée électorale a surtout été rythmée par le cauchemar constitué par la perte - les uns après les autres - de sièges considérés comme sûrs, et qui étaient fidèles au Labour pour certaines depuis toujours, pour d'autres au moins depuis les années 1930 et/ou qui n'avaient jamais élu de député conservateur de leur histoire jusqu'au 12 décembre 2019: Ashfield, Bishop Auckland, Blyth Valley, Bolsover, Darlington, Great Grimsby, Leigh, Rother Valley, Sedgefield, Stoke, West Brom, Workington, etc ${ }^{9}$. Dans ces territoires de vieille industrie (mines, sidérurgie, manufactures), cœurs de communautés ouvrières et populaires, la baisse enregistrée par le parti travailliste a été notoirement plus importante qu'en moyenne nationale. Ainsi par exemple dans le Yorkshire ou le nord-est le vote Labour a reculé respectivement de 10 et 13 points, contre 6 en moyenne à Londres ou dans le sud-est anglais ${ }^{10}$. Au final alors qu'en 2017 sur un total de 158 circonscriptions - soit un quart environ du total de la représentation britannique - correspondant à ce 'red wall' anglais, le Labour en avait gagné 117 - contre 40 aux Conservateurs - la soirée électorale de 2019 a vu le basculement d'une trentaine de sièges travaillistes directement vers le parti de Boris Johnson : la représentation travailliste est passée à 88 sièges, quand dans le même temps les Conservateurs gagnaient 28 sièges pour un total de $68^{11}$.

\section{Le leadership en question(s)}

18 Si l'on s'interroge sur les racines de cette déroute, plusieurs éléments explicatifs peuvent être avancés qui, combinés, ont contribué à produire ce résultat historique. Si l'importance du vote tactique ainsi que le poids de la presse populaire proconservatrice - des constantes dans les élections britanniques - ne sont pas à négliger, il semble que les principales raisons de l'échec travailliste sont à rechercher dans les enjeux liés au leadership, au programme et à la stratégie électorale, ainsi qu'à la question du Brexit.

19 Leader du parti travailliste depuis septembre 2015 Jeremy Corbyn a attaqué la campagne électorale législative de 2019 avec le pire indice de satisfaction enregistré pour un leader de l'opposition depuis 1970 ${ }^{12}$. En septembre 2019 76\% des Britanniques se disaient "mécontents» de Corbyn comme leader de l'opposition, contre 16\% seulement de « satisfaits ", soit un différentiel record de -60. Dans le camp travailliste il faut remonter à Michaël Foot en août 1982 pour trouver un score comparable - 13\% de satisfaits contre $69 \%$ de mécontents, soit un différentiel de -56 . Par ailleurs à la question : «Jeremy Corbyn a-t-il fait un bon travail sur la gestion du Brexit ? », 77\% des 
Britanniques répondaient «non » contre $14 \%$ seulement qui répondaient « oui » - soit un différentiel de -63. Et les sympathisants travaillistes avaient également un avis globalement négatif sur ce point $-34 \%$ de « oui », contre $48 \%$ de «non ».

D'autres enquêtes ont également montré que l'électorat britannique dans son ensemble portait un jugement sévère sur l'équation personnelle du leader travailliste. A la veille des élections si les qualités attribuées à Corbyn portaient avant tout sur son honnêteté foncière et sa capacité à comprendre les problèmes du pays - sans pour autant recevoir une adhésion majoritaire - il ne recueillait un avis favorable que d'un Britannique sur cinq environ sur des attributs comme l'aptitude au leadership ou la capacité à gérer des crises, la personnalité ou la qualité de jugement, la confiance inspirée ou l'aptitude à représenter le Royaume-Uni sur la scène mondiale (cf. tableau 5).

Tableau 5 : Les qualités attribuées à Jeremy Corbyn par l'électorat britannique (en \% de réponses positives)

$45 \%$ Corbyn is out of touch with ordinary people

$43 \%$ Corbyn understands the problems facing Britain

$37 \%$ Corbyn is more honest than most politicians

$36 \%$ Corbyn is patriotic

$27 \%$ Corbyn is more style than substance

$25 \%$ Corbyn has sound judgement.

$25 \%$ Corbyn has got a lot of personality

$21 \%$ Corbyn is a capable leader

$20 \%$ Corbyn is good in a crisis

$20 \%$ Corbyn is a good representative of Britain on the world stage

$19 \%$ Corbyn gives confidence in Britain's future

Source Ipsos Mori, 20/09/2019

Pendant la campagne électorale Corbyn a dû faire face à une série de critiques personnelles, largement relayées par la presse, en relation notamment avec ses prises de position anciennes et son pacifisme affiché en matière de politique étrangère ${ }^{13}$. D'une part suite à son positionnement sur la question palestinienne et son soutien au Hamas, la question de son antisémitisme supposé a été une nouvelle fois mis sur le devant de la scène. Au-delà de ses choix individuels c'est plutôt l'idée que Corbyn avait laissé prospérer une forme d'antisémitisme latent au sein du Labour pendant son leadership, et la perception d'un refus de Corbyn de condamner clairement et spécifiquement l'antisémitisme comme une forme particulière de discrimination, qui a pu éloigner - en dehors de la communauté juive - certains électeurs éduqués et/ou aisés d'un vote Labour, à Londres notamment. D'autre part de par ses prises de position sur la question irlandaise Corbyn a été accusé de complaisance à l'égard de l'IRA et du terrorisme en général, alimentant l'image d'un leader défaillant au plan du patriotisme, un argument non négligeable aux yeux de nombreux électeurs, notamment âgés, issus des milieux populaires et/ou partisans du Leave. Sur le terrain les retours du canvassing rapportés off the record une fois le résultat final connu ont majoritairement fait état d'un rejet très important de la personne de Corbyn, de nombreux candidats du Labour attestant d'une « impopularité monumentale » de leur leader, fortement préjudiciable à la cause travailliste ${ }^{14}$.

D'une manière générale Jeremy Corbyn a été perçu par l'opinion britannique comme défaillant sur la question du leadership - un avis sans doute renforcé par le refus de 
Corbyn de se positionner personnellement sur la question du Brexit tout au long de la campagne - et nettement moins crédible que Boris Johnson pour occuper la fonction de Premier Ministre. Ainsi au moment du vote à la question «Lequel des deux ferait un meilleur Premier Ministre? ", 49\% des Britanniques répondaient Johnson, contre 31\% qui choisissaient Corbyn. Et si les deux leaders étaient rejetés à part sensiblement égale par les électeurs écologistes et libéraux-démocrates, Johnson recevaient le soutien de 75\% des électeurs du Brexit party et surtout 95\% des électeurs conservateurs, alors que Corbyn ne recueillait « que » $76 \%$ de soutien dans son propre camp. Donc si l'adhésion à Johnson dans l'électorat conservateur était quasi unanime, elle était assez nettement plus forte que celle à Corbyn chez les Travaillistes.

Tableau 6 : Le meilleur Premier Ministre selon les préférences de l'électorat

Réponse à la question : qui ferait selon vous le meilleur Premier Ministre?

\begin{tabular}{|l|l|l|l|l|l|l|l|}
\hline & $\begin{array}{l}\text { Ensemble } \\
\text { des } \\
\text { électeurs }\end{array}$ & $\begin{array}{l}\text { Electeurs } \\
\text { Conser- } \\
\text { vateurs }\end{array}$ & $\begin{array}{l}\text { Electeurs } \\
\text { Travallistes }\end{array}$ & $\begin{array}{l}\text { Electeurs } \\
\text { Lib- } \\
\text { Dems }\end{array}$ & $\begin{array}{l}\text { Electeurs } \\
\text { SNP }\end{array}$ & $\begin{array}{l}\text { Electeurs } \\
\text { Greens }\end{array}$ & $\begin{array}{l}\text { Electeurs } \\
\text { Brexit } \\
\text { party }\end{array}$ \\
\hline Corbyn & 31 & 0 & 76 & 26 & 51 & 28 & 4 \\
\hline Johnson & 49 & 95 & 3 & 19 & 8 & 19 & 75 \\
\hline
\end{tabular}

Source : Lord Ashcroft polls décembre 2019

Au final en dépit de toutes les critiques pouvant être adressées à Johnson, son équation personnelle, à la fois dynamique et entrainante, a pu contraster avec celle de Corbyn et jouer en faveur de la majorité au pouvoir. Les attentes de l'opinion d'un leadership fort pour éclairer la marche à suivre et/ou accomplir le processus du Brexit, qui avaient joué un rôle important en 2017 à l'encontre de Theresa May, ont cette fois joué en faveur de Johnson et au détriment de Corbyn.

\section{Les incertitudes du programme et de la stratégie électorale}

Un autre élément explicatif de la déroute du Labour est à rechercher du côté de son programme et de sa stratégie électorale ${ }^{15}$. Pour les élections de 2019 , le manifeste du parti travailliste intitulé 'It's time for a real change', riche de plus de cent pages, se présentait comme un catalogue extrêmement détaillé de propositions de réformes tous azimuts, d'inspiration sociale, écologiste et dirigiste, très engagées à gauche ${ }^{16}$ : la renationalisation de nombreux secteurs économiques clés comme les chemins de fer, la poste, l'eau ou l'énergie ; la suppression du Universal Credit ; l'introduction d'un National Care Service, gratuit pour les personnes âgées; l'investissement massif dans la révolution verte ; la création d'un million d'emplois dans le changement climatique ; la constitution d'un service d'éducation national ; la hausse significative du budget de la santé ; la construction annuelle de 150.000 logements sociaux ; l'augmentation sensible du revenu minimum; la création de nouveaux impôts - notamment sur les résidences secondaires, les droit de succession ou les droits d'inscription dans des écoles privées ; 
l'abolition des droits de scolarité universitaires ; la gratuité des transports en bus pour les moins de 25 ans; la gratuité généralisée des connexions haut débit, constituaient certaines des mesures phares mises en avant par les Travaillistes lors de la campagne ${ }^{17}$. Par son caractère radical et son objectif de remettre en question non seulement les politiques d'austérité des récents gouvernements conservateurs mais aussi le modèle économique néo-libéral en vigueur au Royaume-Uni depuis l'avènement du thatchérisme ${ }^{18}$, ce programme n'était pas sans écho avec celui présenté par le parti travailliste de Michael Foot lors des élections de $1983^{19}$. Si, contrairement à 1983, le manifeste travailliste ne contenait pas de propositions chocs susceptibles de cliver le parti ${ }^{20}$, le programme de 2019 a pu apparaitre à certains d'électeurs comme faisant écho, par son approche idéologique, au climat d'affrontement social des années 1980 et/ou à une époque révolue. Par ailleurs si un certain nombre des promesses prises individuellement - comme par exemple la renationalisation des chemins de fer ou la baisse des droits universitaires - bénéficiait d'un soutien majoritaire dans l'électorat, le programme dans son ensemble pouvait être considéré comme exagérément prolifique et/ou financièrement irréaliste.

$\mathrm{Au}$ soir de la défaite certains dirigeants proches de Jeremy Corbyn ont reconnu le caractère trop ambitieux du projet. John Lansman, fondateur de Momentum, le groupe de soutien à Corbyn au sein du Labour, déclarait par exemple: 'The manifesto was too detailed and too long. It was a programme for 10 years, not for government' ${ }^{\prime 21}$. D'autres élus travaillistes ont également pointé du doigt le côté "attrape-tout " et l'absence de cohérence globale du programme. Ainsi Chi Onwurah, députée de Newcastle et ministre de la stratégie industrielle au sein du Cabinet fantôme, décrivait le manifeste du Labour comme 'a huge Christmas tree of policy promises' tandis que Phil Wilson, battu à Sedgefield dans une circonscription historiquement travailliste autrefois détenue par Tony Blair, déclarait :

You could find some policy item you agreed with but the manifesto has got to add up to something coherent...The impression I got is someone writing the manifesto was sitting in an office with a queue of pressure groups outside coming in saying: can you add this to the list? Which we did without any thought and without being rational

La question de la crédibilité du programme et de sa faisabilité économique est aussi venue rapidement sur le devant de la scène, en écho aux retours critiques enregistrées sur le terrain pendant la campagne. Chi Onwurah déclarait par exemple :

\footnotetext{
We had a manifesto crammed with transformative policies that people didn't believe in. We did not have a credible response to people's pain - not only the deindustrialisation of the past, but also the fears associated with globalisation, technology and financial speculation. They all create anxiety about where good jobs will come from..... Our policies seemed to be about a better division of the pie when people didn't believe in the pie any more' ${ }^{\prime 2}$.
}

C'est aussi le sens des critiques virulentes d'Alan Johnson, ancien Home secretary dans le gouvernement Brown et proche de Blair, qui déclarait au soir des résultats: 'Labour tried to fool the public into thinking that money was no object, which made the manifesto look like the wish-list of a pressure group rather than a serious plan for a potential party of government'. Ce à quoi faisaient écho certains éditoriaux politiques dans la presse de centre gauche, tel ce commentaire dans the Guardian : 
For the last four years, Labour has been in thrall to the notion that it's better to have a manifesto you can feel proud of, a programme that calls itself radical, than to devise one that might have a chance of winning. Some even argued that, "win or lose", Corbyn achieved much simply by offering a genuinely socialist plan - in contrast with Labour's 1997 offer, which was so boringly modest and incremental. ${ }^{23}$

31 L'éventail de ces critiques à l'encontre de la stratégie électorale travailliste doit évidemment s'interpréter à l'aune des fortes tensions existant entre les dirigeants du Labour, et du positionnement des différentes tendances internes dans la perspective de l'après-Corbyn. Si la gauche radicale ou orthodoxe du Labour a pu concéder le «trop plein » du programme travailliste ou le manque de « coherent narrative» de la campagne comme éléments explicatifs de la défaite ${ }^{24}$, les modérés et les Blairistes n'ont eu de cesse d'insister au soir de la défaite sur le caractère radical, passéiste ou déconnecté de la réalité du manifeste.

\section{Le Brexit conundrum}

La question du Brexit, qui a complètement dominé la scène politique britannique pendant trois ans et demi à partir du référendum de juin 2016, a également beaucoup pesé à l'occasion de ces élections législatives, essentiellement au détriment des Travaillistes. Face au message simple et fort de Boris Johnson visant à «réaliser le Brexit » -'Get Brexit done' - et donc clore définitivement les débats sur le sujet et tourner la page du processus de retrait de l'Union Européenne - le parti travailliste a indiqué qu'en cas de victoire, il souhaitait renégocier un accord avec l'UE dans un délai de trois mois, puis soumettre trois mois après au plus tard ce nouvel accord aux Britanniques par le biais d'un référendum clairement impératif qui proposerait aussi comme alternative le maintien dans l'UE; et qu'il s'engageait à mettre en œuvre la décision finale du peuple britannique, sans prendre position à l'occasion de la nouvelle consultation.

Ce positionnement du parti travailliste tentait de résoudre les tensions internes au Labour sur ce sujet - notamment sur le principe de l'organisation d'un deuxième référendum - en essayant de satisfaire les deux camps de sa coalition électorale tout à la fois : d'une part les Remainers - majoritaires chez les membres et sympathisants du parti et correspondant à un électorat majoritairement jeune, urbain, diplômé et/ou multiculturel; et d'autre part les Leavers - quantitativement nettement minoritaires mais symboliquement importants dans les bastions ouvriers travaillistes du red wall anglais et gallois, et correspondant à un électorat majoritairement âgé, populaire et issu des villes moyennes et des territoires ruraux et/ou périphériques ${ }^{25}$.

Dans la pratique ce positionnement médian a été globalement jugé comme une formulation compliquée et floue, et a plutôt eu l'effet de mécontenter les partisans des deux camps : pour les Leavers le choix travailliste tendait à remettre en cause le vote démocratique majoritaire exprimé par 17,4 millions de Britanniques à l'occasion du référendum de 2016; tandis que les Remainers jugeaient la position travailliste beaucoup trop timide, d'autant que le leadership a refusé systématiquement de s'engager sur l'issue, comme le débat télévisé entre Corbyn et Johnson a pu notamment le confirmer. In fine l'enjeu du Brexit s'est révélé extrêmement compliqué à gérer pour les Travaillistes, tandis que dans le même temps les Conservateurs faisaient clairement 
le choix de revendiquer le "Leave" afin de recueillir le soutient maximal de ses supporters, tout en offrant une solution définitive à la partie de l'électorat lassée par plus de trois ans de débats interminables sur l'enjeu européen.

Sur le terrain les différentes enquêtes ont montré que le Brexit a constitué l'un des deux enjeux majeurs du scrutin, avec l'avenir du système de santé. Les différents électorats ont tous classé ces deux thèmes en tête de leurs priorités au moment du vote, les électeurs travaillistes et SNP privilégiant le NHS, tandis que les autres électeurs plaçaient le Brexit en numéro un, soit afin de le voir se réaliser - pour les électeurs conservateurs ou du Brexit party - soit pour le stopper - dans le cas des électeurs libéraux-démocrates ${ }^{26}$. Pendant la campagne la stratégie travailliste a notamment consisté à essayer d'attirer le débat vers la question du NHS et les enjeux sociaux, afin de contrebalancer l'impact du Brexit. Cependant le discours populiste et "one nation" de Johnson et ses promesses de fin d'austérité budgétaire et d'investissement significatif dans le NHS, ont réussi à neutraliser en grande partie cet enjeu.

$\mathrm{Au}$ final si les partis britanniques favorables au Remain ont recueilli un peu plus de 17 millions de voix -contre un peu moins de 15 millions pour l'ensemble des formations pro-Leave - le transfert des électeurs des deux camps entre les deux grands partis a été nettement préjudiciable aux Travaillistes (cf. Tableau 7).

Tableau 7 : Le vote des Remainers et des Leavers de 2016 lors des élections législatives de 2019 et 2017

\begin{tabular}{|c|c|c|c|c|c|c|c|c|}
\hline & & $\begin{array}{l}\text { Vote } \\
\text { Travailliste }\end{array}$ & $\begin{array}{l}\text { Vote } \\
\text { Conser- } \\
\text { vateur }\end{array}$ & $\begin{array}{l}\text { Vote } \\
\text { Lib- } \\
\text { Dems }\end{array}$ & $\begin{array}{l}\text { Vote } \\
\text { SNP }\end{array}$ & $\begin{array}{ll}\text { Vote } & \\
\text { UKIP ou } \\
\text { Brexit } \\
\text { Party }\end{array}$ & $\begin{array}{l}\text { Vote } \\
\text { Greens }\end{array}$ & $\begin{array}{l}\text { Autres } \\
\text { votes }\end{array}$ \\
\hline \multirow{2}{*}{$\begin{array}{l}\text { Elections } \\
2019\end{array}$} & $\begin{array}{l}\text { Remain } \\
2016\end{array}$ & 47 & 20 & 21 & 6 & - & 4 & 1 \\
\hline & $\begin{array}{l}\text { Leave } \\
2016\end{array}$ & 16 & 73 & 3 & 2 & 4 & 2 & 1 \\
\hline \multirow{2}{*}{$\begin{array}{l}\text { Elections } \\
2017\end{array}$} & $\begin{array}{l}\text { Remain } \\
2016\end{array}$ & 51 & 25 & 14 & 6 & - & 3 & 2 \\
\hline & $\begin{array}{l}\text { Leave } \\
2016\end{array}$ & 25 & 60 & 4 & 2 & 6 & 2 & 1 \\
\hline
\end{tabular}

Source : Lord Ashcroft polls, 2019

Ainsi aux élections législatives de 2019, les Travaillistes ont récupéré environ $47 \%$ du vote Remain 2016 et 16\% du vote Leave 2016. Par comparaison les Conservateurs ont recueilli environ $20 \%$ du vote Remain 2016 et $73 \%$ du vote Leave 2016. Le vote Remain s'est trouvé assez dispersé - les Libs-Dems en recueillant notamment 21\%, le SNP 6\% et les Verts $4 \%$ - et les Travaillistes n'ont pas réussi à recueillir le soutien majoritaire moins d'un votant sur deux - chez cet électorat. A l'opposé les électeurs pro-Leave se sont très majoritairement - pratiquement trois sur quatre - portés vers le parti conservateur. Et bien que se focalisant prioritairement sur les électeurs pro-Leave, le 
parti conservateur a réussi à recueillir une partie non négligeable du vote Remain - $20 \%$, soit un sur $\mathrm{cinq}^{27}$. Au final sur les 401 circonscriptions supposées avoir voté Leave lors du référendum de 2016, les Conservateurs réussirent en 2019 à en gagner 292, soit 73\%. Dans le même temps les Travaillistes ne gagnèrent que $41 \%$ de l'ensemble des circonscriptions sensées avoir voté Remain - 95 sièges sur 231 .

L'évolution par rapport aux précédentes élections de 2017 est également éclairante (cf. tableau 7). Ainsi en 2017 le Labour avait récupéré 51\% des Remainers - soit plus 4 pts par rapport à 2019 - et un quart des Leavers - plus 9 par rapport à 2019 - tandis que le parti Conservateur de Theresa May recueillait un quart des Remainers (+ 5 par rapport à 2019) et $60 \%$ des Leavers (-13 par rapport à 2019). On voit donc qu'entre 2017 et 2019 les Travaillistes ont perdu en chemin des électeurs des deux camps et - si l'on considère ces derniers globalement équivalents au plan quantitatif - deux fois plus de Leavers que de Remainers, tandis que les Conservateurs perdaient des Remainers - en nombre à peu près égal à ceux perdus par les Travaillistes - tout en gagnant beaucoup d'électeurs pro-Leave.

D'autres enquêtes de terrain ont corroboré ces analyses ${ }^{28}$. Ainsi sur les 54 sièges perdus par les Travaillistes en 2019 directement au profit des Conservateurs, 52 avaient voté Leave en 2016. Plus généralement il a été montré que si le vote travailliste en 2019 avait globalement diminué par rapport à 2017, cette baisse était plus forte dans les circonscriptions qui avaient voté Leave plutôt que dans celles pro-Remain, et que ce recul était d'autant plus important que le vote Leave en 2016 avait été conséquent : ainsi une baisse de 10,4\% en moyenne a été enregistrée pour le score du Labour dans les sièges où au moins $60 \%$ de l'électorat avaient voté Leave. Dans le même temps le score du parti conservateur progressait de 6,1\% par rapport à 2017 - soit cinq fois plus que sa moyenne nationale - dans ces circonscriptions où le vote Leave 2016 était au moins égal à $60 \%{ }^{29}$.

Cette désaffection d'une partie non négligeable de l'électorat sur l'enjeu du Brexit - et notamment des partisans du Leave - préjudiciable au Labour en 2019, a pu répondre à trois motivations différentes et complémentaires: d'une part la lassitude par rapport aux débats sur le Brexit et la volonté de clore la question, ce qui a fait basculer certains Remainers dans le camp conservateur après avoir voté travailliste en 2017 ; d'autre part, le positionnement du leadership travailliste sur le sujet, articulé autour des notions de renégociation et de neutralité en même temps, jugé par l'opinion au mieux compliqué et équivoque, au pire intenable et irresponsable ; enfin l'idée d'organiser une nouvelle consultation sur le sujet - contestée par $80 \%$ des Leavers - et le non-respect du résultat de juin 2016, assimilé à un déni de démocratie et significatif - aux yeux des Leavers prioritairement - de l'absence d'écoute des dirigeants travaillistes vis-à-vis de leur base populaire.

\section{Le débat 'It was Brexit' vs 'It was Corbyn' et ses limites}

$41 \mathrm{Si}$ les trois causes principales de la déroute travailliste semblent difficilement contestables, quelle est la part respective de chacune d'entre elles dans le résultat final? Dès le soir des élections la polémique s'est engagée sur ce point dans les rangs travaillistes : d'un côté Jeremy Corbyn et ses supporters ont insisté sur le rôle essentiel joué par le Brexit comme enjeu singulier et exceptionnel ayant contribué à polariser l'électorat britannique et surtout occulter le débat sur l'agenda socio-économique et 
des propositions travaillistes globalement populaires dans l'opinion. D'un autre côté la critique des anti-Corbynistes s'est focalisée sur le leadership et le programme du Labour, en insistant sur l'impopularité record de Corbyn enregistrée sur le terrain pendant la campagne, et sur le caractère irréaliste et/ou «déconnecté » du message socialiste proposé à l'électorat ${ }^{30}$.

De fait ces trois facteurs sont complémentaires et ont interagi entre eux comme l'ont montré plusieurs enquêtes sorties des urnes ou réalisées dans la foulée des élections. Ainsi dans l'enquête de grande envergure conduite par Lord Ashcroft auprès de l'électorat britannique ${ }^{31}$, il apparait que pour l'ensemble des électeurs ayant voté travailliste, la principale motivation était de loin la volonté d'empêcher l'installation d'un nouveau gouvernement conservateur, suivie par l'idée que les propositions travaillistes seraient bénéfiques pour la gestion du NHS et les services publics, beaucoup plus que le souhait de voir Jeremy Corbyn devenir Premier Ministre ou la conviction que le Labour constituerait un gouvernement plus compétent, qui ressortent comme des facteurs nettement secondaires ${ }^{32}$. La crédibilité personnelle de Jeremy Corbyn à occuper la fonction de Premier Ministre, et celle de la direction travailliste à gouverner le pays, ne semblaient pas une donnée fondamentale aux propres yeux de l'électorat du Labour.

Par ailleurs si l'on s'intéresse aux motivations des électeurs ayant voté travailliste en 2017 mais ayant déserté le Labour en 2019, les principales raisons avancées pour justifier ce changement ont trait prioritairement à Corbyn - et au refus de favoriser son accession au 10 Downing Street - au programme travailliste - jugé irréaliste - et à l'identité du Labour, perçue comme loin des aspirations de l'électorat. Dans le détail les votants étant passés d'un vote travailliste en 2017 à un vote conservateur en 2019 intègrent aussi la question du Brexit comme une motivation très importante de leur choix électoral, pratiquement à égalité avec le rejet de Corbyn (cf. Tableau 8). Pour certains électeurs à la fois proches des conservateurs et pro-Remain, la crainte d'un gouvernement dirigé par Jeremy Corbyn l'a clairement emporté sur le désir de voir le Royaume-Uni rester dans l'Union Européenne.

Tableau 8 : Les motivations des électeurs ayant abandonné le parti travailliste en 2019

\begin{tabular}{|c|c|c|}
\hline $\begin{array}{l}\text { Ensemble des électeurs ayant } \\
\text { voté travailliste en } 2017 \text { mais } \\
\text { pas en } 2019\end{array}$ & \begin{tabular}{|lrr} 
Electeurs & ayant & voté \\
travailliste & en 2017 & et \\
conservateur en 2019 &
\end{tabular} & $\begin{array}{l}\text { Electeurs ayant voté } \\
\text { travailliste en } 2017 \text { et } \\
\text { libéraux-démocrates en } 2019\end{array}$ \\
\hline $\begin{array}{l}\text { Je ne voulais pas que Jeremy Corbyn } \\
\text { devienne Premier Ministre } 53 \%\end{array}$ & $\begin{array}{l}\text { Je ne voulais pas que Jeremy } \\
\text { Corbyn devienne Premier Ministre } \\
75 \%\end{array}$ & $\begin{array}{l}\text { Je ne voulais pas que Jeremy } \\
\text { Corbyn devienne Premier Ministre } \\
\mathbf{5 1 \%}\end{array}$ \\
\hline $\begin{array}{l}\text { Je n'ai pas pensé que le Labour } \\
\text { pourrait réaliser les promesses faites } \\
\mathbf{4 0 \%}\end{array}$ & $\begin{array}{l}\text { Je voulais que le Brexit se réalise } \\
\text { et ai voté pour faire en sorte que } \\
\text { cela se } \\
\text { produise } 73 \%\end{array}$ & $\begin{array}{l}\text { Je n'ai pas pensé que le Labour } \\
\text { pourrait réaliser les promesses } \\
\text { faites } \mathbf{3 6 \%}\end{array}$ \\
\hline $\begin{array}{l}\text { Le parti travailliste ne me semble } \\
\text { plus représenter des gens comme } \\
\text { moi } 37 \%\end{array}$ & $\begin{array}{l}\text { Je n'ai pas pensé que le Labour } \\
\text { pourrait réaliser les promesses } \\
\text { faites } 62 \%\end{array}$ & $\begin{array}{l}\text { Un autre parti semblait plus près } \\
\text { de mes valeurs et de ma vision de } \\
\text { la vie } 35 \%\end{array}$ \\
\hline
\end{tabular}




\begin{tabular}{|l|l|l|}
\hline $\begin{array}{l}\text { Je voulais que le Brexit se réalise et } \\
\text { ai voté pour faire en sorte que cela } \\
\text { se produise } \mathbf{3 0 \%}\end{array}$ & $\begin{array}{l}\text { Le parti travailliste ne me semble } \\
\text { plus représenter des gens comme } \\
\text { moi } \mathbf{6 1 \%}\end{array}$ & $\begin{array}{l}\text { Aucune } \\
\text { de ces raisons } \mathbf{2 8 \%}\end{array}$ \\
\hline $\begin{array}{l}\text { Je n'ai pas aimé les politiques } \\
\text { promises par le parti travailliste } \\
\mathbf{2 6 \%}\end{array}$ & $\begin{array}{l}\text { Je n'ai pas aimé les politiques } \\
\text { promises par le parti travailliste } \\
\mathbf{4 9 \%}\end{array}$ & $\begin{array}{l}\text { Le parti travailliste ne me semble } \\
\text { plus représenter des gens comme } \\
\text { moi } \mathbf{2 4 \%}\end{array}$ \\
\hline
\end{tabular}

Source : Lord Ashcroft, Diagnosis of the defeat, Labour's turn to smell the coffee, Février 2020

Concernant l'attractivité du programme travailliste présenté à l'occasion de ces élections, Jeremy Corbyn et ses partisans ont estimé avoir, en dépit de la lourde défaite enregistrée, gagné le débat politique contre l'austérité et en faveur du changement social et de politiques fortement interventionnistes et redistributives ${ }^{33}$. Cet argument s'appuie notamment sur le fait que, proposées individuellement, la plupart des promesses $\mathrm{du}$ programme travailliste étaient relativement populaires auprès de l'électorat : il en est ainsi par exemples des propositions de nationalisation des chemins de fer, de l'eau ou du secteur de l'énergie, soutenues respectivement par $57 \%, 52 \%$ ou $53 \%$ de l'électorat. Cependant les Britanniques se révélaient beaucoup plus critiques sur l'idée même de renationalisation et sur le manifeste travailliste dans son ensemble ${ }^{34}$. Ce paradoxe apparent peut s'expliquer par l'effet cumulatif de la somme des promesses, qui a pu conduire certains électeurs à considérer le programme travailliste pris globalement comme excessif et irréaliste - 'the pie in the sky' - et d'autre part par la conviction que la direction travailliste ne possédait pas la crédibilité et la compétence nécessaires pour mettre en œuvre un manifeste aussi exhaustif, ce qui est une critique directe à l'encontre de Corbyn et de la direction travailliste. L'impopularité personnelle rencontrée par le leader travailliste a pu ainsi impacter de façon négative le jugement global de l'électorat sur le programme du Labour, et représenter un facteur contribuant à la baisse de l'attractivité des Travaillistes lors des élections.

Au-delà des incertitudes pesant sur l'aptitude du Labour à réaliser son programme, ces enquêtes reflètent de nombreuses interrogations et critiques sur l'identité politique actuelle du parti travailliste, et de sa capacité à représenter son électorat traditionnel issu de la classe ouvrière et des milieux populaires. Tout autant voire plus que les enjeux liés au leadership et à la question du Brexit, c'est le sentiment que le Labour de 2019 ne parlait plus en leurs noms, ne comprenait plus leurs aspirations, ne partageait plus leurs valeurs et était déconnecté de la réalité sociale et individuelle quotidienne vécue dans les communautés et les territoires comme ceux du 'red wall', qui a poussé de nombreux électeurs à se désengager et rompre le lien électoral ancien établi avec le Labour, au profit des Conservateurs.

En ce sens il s'agit d'un phénomène de désalignement qui n'a certes pas commencé en 2019, mais que l'enjeu du Brexit et les incertitudes entourant le leadership travailliste ont ponctuellement accentué. 


\section{Conclusion : l'implosion de sa coalition électorale et la crise existentielle du parti travailliste}

L'élection de 2019 a vu se disloquer la coalition électorale historique soutenant le parti travailliste, suite à la désaffection d'une partie importante de son électorat traditionnel, ouvrier et populaire. Si le Labour a obtenu des résultats satisfaisants dans les grandes agglomérations ou les villes universitaires, s'il est attractif auprès des catégories jeunes ou diplômées et semble avoir résolu son problème avec les classes moyennes éduquées du sud anglais, il s'est aliéné ses anciens bastions industriels des Midlands et du nord anglais, sans parler du Central Belt écossais. Le pari de Corbyn, selon lequel un message politique caractérisé par une forte inspiration sociale et articulé autour du conflit de classe et de la mobilisation collective pourrait séduire cet électorat et l'emporter sur les enjeux individuels et identitaires - à la fois émotionnels et d'ordre national - mobilisés par le Brexit, n'a pas fonctionné. Bien au contraire le Brexit a accéléré un réalignement électoral de long terme ${ }^{35}$.

En ce sens l'élection de 2019 est très différente des précédentes déroutes travaillistes de grande ampleur enregistrées dans les années $1980^{36}$. Ainsi en 1983 le Labour avait été balayé dans le sud du pays par la vague thatchérienne néo-conservatrice, mais il avait largement remporté le soutien de l'électorat populaire, notamment dans ses bastions industriels du 'red wall', dans le nord anglais et la périphérie écossaise et galloise, inaugurant une longue décennie électorale caractérisée par le 'north-south divide'.

Aux élections de 2010, après treize années de gouvernements New Labour qui avaient prioritairement cherché - et en partie obtenu - le soutien des classes moyennes urbaines et éduquées et mis un terme au 'southern discomfort' des Travaillistes, ces derniers, malgré la défaite, conservaient toujours une avance conséquente sur le parti conservateur dans les circonscriptions populaires, même si celles-ci, considérées comme définitivement acquises au Labour, pouvaient s'estimées négligées par Londres et connaissaient une forme d'apathie électorale. Cependant au cours de la dernière décennie cet avantage concurrentiel historique du Labour auprès de l'électorat ouvrier s'est progressivement érodé pour appartenir désormais au passé : après la perte de la 'red Clydeside' écossaise, bastion historique du mouvement ouvrier britannique, 2019 a été marquée par les craquements du 'mur' gallois et l'effondrement du 'red wall' anglais ${ }^{37}$.

C'est donc à une crise existentielle majeure, en lien avec l'identité originelle du Labour liée à la défense des communautés ouvrières et des milieux populaires, à laquelle la défaite historique de 2019 confronte aujourd'hui le parti travailliste.

Gilles Leydier est professeur de civilisation britannique contemporaine à l'Université de Toulon et membre du Laboratoire Babel (EA 2649). Spécialiste de la vie politique britannique, il a publié de nombreux travaux sur les institutions, le système partisan et la sociologie électorale, notamment Partis et élections au Royaume-Uni depuis 1945 (2004) et Le Royaume-Uni à l'heure de la coalition (2014). Il est directeur de la publication de la revue l'observatoire de la société britannique, et président du Centre de recherches en civilisation britannique (CRECIB). 


\section{BIBLIOGRAPHIE}

Bale, Tim, What does Brexit mean for the UK's party political kaleidscope ?, The UK in a changing Europe, 10/02/2020.

Curtice, John, What's behind the Conservative victory?, The UK in a changing Europe, 13/12/2020.

Cutts, D., Goodwin, M., Heath, O. \& Surridge, P., Brexit, the 2019 General Election and the Realignment of British Politics, The Political Quarterly, 19/02/2020.

Economist (The), Victory for Boris Johnson's all-new Tories, 13 /12/2019.

Ford, Robert, Britain's new political landscape: what the voting numbers tell us, The Guardian, 15/12/ 2019.

Guardian (The), Labour's defeat: an existential crisis with no easy solution, 15/12/2019.

Lawrence, John, Labour and the Culture Wars of Modern Politics, The Political Quarterly, 19/02/2020.

Parry, Richard, England votes for a break from politics, Centre on Constitutional Change, 19/12/2019.

McCrone, David, Keeping Perspectives, Centre on Constitutional Change, 16/12/2019.

Labour Party (The), It's time for a real change, Electoral Manifesto 2019.

Lord Ashcroft, Diagnosis of the defeat, Labour's turn to smell the coffee, 10/02/ 2020.

Menon, A. \& Wager, A. Labour's Brexit dilemma, 2019 Brexit and public opinion, The UK in a changing Europe.

Proctor, Kate, Five reasons why Labour lost the election, The Guardian, 14/12/2019.

Report Brexit, The manifestos uncovered', The UK in a changing Europe, décembre 2019.

Sturridge, Paula, Labour lost its leavers while Tory remainers stayed loyal, The UK in a changing Europe,13/12/2019.

Wager, Alan, The Labour Party's electoral challenges, The UK in a changing Europe, 02/03/2020.

\section{NOTES}

1. Voir par exemple Robert Ford, Britain's new political landscape: what the voting numbers tell us, The Observer, 15/12/ 2019 ; The Guardian view on Labour's defeat: an existential crisis with no easy solution, $15 / 12 / 2020$.

2. L'âge à partir duquel l'électeur est plus susceptible de voter conservateur plutôt que travailliste, a cependant nettement avancé entre 2017 et 2019, passant de 47 à 39 ans. Cf. Adam McDonnell \& Chris Curtis, How Britain voted at the 2019 general election, Yougov, 17/12/2019.

3. Mais entre 2017 et 2019 les Travaillistes ont régressé dans chacune de ces trois catégories. Cf. How Britain voted at the 2019 general election, Yougov, opus cité.

4. Richard Parry, England votes for a break from politics, Centre on Constitutional Change 19/12/ 2019.

5. Voir aussi la contribution de Stéphanie Bory dans ce volume.

6. Voir aussi les contributions d'Edwige Camp-Pietrain et de Fiona Simpkins dans ce volume.

7. David McCrone, Keeping Perspectives, Centre on Constitutional Change, 16/12/2019.

8. Boris Johnson thanks North for trusting Tories, BBC, 14/12/2019. 
9. Daniel Wainwright, How Labour's 'red wall' turned blue, BBC, 13/12/ 2019.

10. John Curtice, What's behind the Conservative victory?, The UK in a changing Europe, 13/12/2019.

11. How the north of England has changed, BBC, 13/12/ 2019.

12. Jeremy Corbyn has lowest leadership satisfaction rating for any opposition leader since 1977, Ipsos Mori, 20/09/2019.

13. Cf. par exemple Corbyn, antisemitism and Brexit: Labour MPs on why they lost, The Guardian, 17/12/2020 ; Brexit and self-inflicted errors buried Labour in this election, The Guardian, 18/12/2020.

14. Cf. par exemple Kate Proctor, Five reasons why Labour lost the election, The Guardian, 14/12/19.

15. Voir aussi dans ce numéro la contribution de Toufik Abdou.

16. It's time for a real change, Labour Party Manifesto 2019.

17. Labour Party manifesto 2019: 12 key policies explained, BBC news, 21/11/2020 ; Labour manifesto: what it says and what it means, The Guardian, 21/11/2019.

18. Cf. par exemple 'Labour will rewrite the rule of the economy...', Introduction de Jeremy Corbyn au manifeste travailliste.

19. Ce programme travailliste de 1983 a été par la suite décrit par certains historiens comme 'the longest suicide note in (British political) history', à la suite des déclarations de Gerard Kaufman, membre du cabinet fantôme travailliste de Michael Foot.

20. Comme c'était le cas en 1983 avec l'engagement à quitter la CEE ou la promesse de désarmement nucléaire unilatéral.

21. Cf. 'Five reasons why Labour lost the elections', The Guardian, 13/12/2019.

22. Chi Onwurah, New message, new voice: how Labour can win back working-class voters, The Guardian, 18/12/2019.

23. Jonathan Freedland, This is a repudiation of Corbynism. Labour needs to ditch the politics of the sect, The Guardian, 13/02/2019.

24. Cf. par exemple Owen Jones, Brexit and self-inflicted errors buried Labour in this election, The Guardian, 18/12/2020 ; et Andrew Murray, Assessing defeat, Tribune, 08/02/2020.

25. Anan Menon \& Alan Wager, A. Labour's Brexit dilemma, 2019 Brexit and public opinion, The UK in a changing Europe.

26. Cf. par exemple Lord Ashcroft polls 2019

27. Paula Sturridge, Labour lost its leavers while Tory remainers stayed loyal, The Guardian, $13 / 12 / 2019$.

28. Cf. par exemple John Curtice, What's behind the Conservative victory, The UK in a changing Europe? 13/12/2019.

29. Chris Hanretty, Labour lost votes in Leave and Remain areas, BBC, 12/12/2020.

30. Voir par exemple Will Hutton, Unions colluded in the fiction that Corbyn's plan was going to win power, The Guardian, 15/12/2020.

31. Lord Ashcroft, Diagnosis of the defeat, Labour's turn to smell the coffee, 10/02/2020.

32. Lord Ashcroft, /2020, opus cite, pp. 9/10.

33. Jeremy Corbyn, We won the argument, but I regret we didn't convert that into a majority of change, The Observer, 14/12/2019.

34. Voir par exemple Labour policies popular, but many want change in direction, The Independent, $14 / 01 / 2020$.

35. David Cutts, Matthew Goodwin, Oliver Heath \& Paula Surridge, Brexit, The 2019 General Election and the Realignment of British Politics, The Political Quarterly, 19/02/2020.

36. John Lawrence, Labour and the Culture Wars of Modern Politics, The Political Quarterly, 19/02/2020.

37. Alan Wager, The Labour Party's electoral challenges, The UK in a changing Europe, $02 / 03 / 2020$. 


\section{RÉSUMÉS}

A l'occasion des élections législatives de décembre 2019, le parti travailliste a connu une défaite surprenante et spectaculaire, la quatrième de rang en une décennie, une des pires de son histoire depuis la Seconde Guerre mondiale. L'objectif de cet article est de replacer ce résultat dans une perspective de long terme, puis de cerner les contours actuels de l'électorat du Labour, enfin d'analyser les racines de sa perte d'attractivité. On s'interrogera notamment sur le rôle joué par le leadership, le programme et la stratégie travaillistes, ainsi que par l'enjeu spécifique du Brexit, afin de prendre toute la mesure de l'échec électoral, révélateur pour le Labour d'une crise identitaire profonde.

In the General Election of December 2019, the Labour Party suffered a surprising and spectacular defeat, the fourth in a row within a decade, one of the worst in its history since WWII. The aim of this article is to put this result in a long-term perspective, identify the current features of the Labour electorate, and finally analyse the roots of Labour's loss of attractiveness. Attention will be given to the role played by Labour's leadership, programme and strategy, as well as to the specific issue of Brexit, in order to take full measure of the electoral failure, which reveals a deep identity crisis.

INDEX

Mots-clés : Parti travailliste, élections législatives de 2019, Jeremy Corbyn, Red wall, Brexit Keywords : Labour Party, 2019 general election, Jeremy Corbyn, Red wall, Brexit

\section{AUTEUR \\ GILLES LEYDIER}

Université de Toulon 